\title{
CORRIGENDUM
}

doi:10.1038/nature07880

\section{MicroRNAs to Nanog, Oct4 and Sox2 coding regions modulate embryonic stem cell \\ differentiation}

Yvonne Tay, Jinqiu Zhang, Andrew M. Thomson, Bing Lim

$\&$ Isidore Rigoutsos

\section{Nature 455, 1124-1128 (2008)}

In this Letter, an incorrect micrograph was inadvertently used in the panel entitled 'Nanog-WT $+S c r$ ' in row 1 of Fig. 3a: the used micrograph was from a different field of view from the 'EV $+S c r$ ' experiment. A representative micrograph from the 'Nanog-WT $+S c r$ ' experiment is presented below. This oversight affects neither the data nor the conclusions of the work presented in the manuscript.

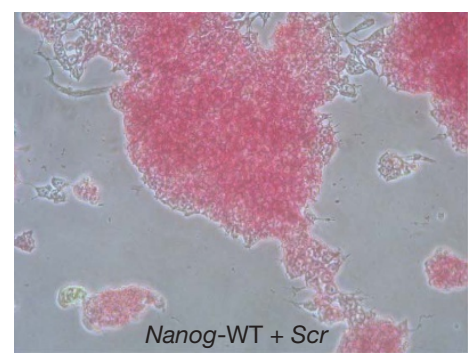

